Conclusions Trainee neonatologists lack confidence in many areas of palliative care provision on neonatal units. This study is the first to generate a neonatal palliative care curriculum specifically for this group of doctors, who are responsible for the care of a significant proportion of children who die. Local priorities and service provision need to be central in the generation of educational curricula and subsequent training programs. This can be supported by improved integration between neonatal and paediatric palliative care networks nationally.

\section{G357 LEARNING FROM BEREAVED FAMILIES TO IMPROVE CHILDREN'S PALLIATIVE CARE}

1,2A Soman, ${ }^{3} \mathrm{~L}$ Maynard. 'Paediatric Palliative Care, Sheffield Children's NHS FT, Sheffield, UK; ${ }^{2}$ Bluebell Wood Children's Hospice, Sheffield, UK; ${ }^{3}$ East Anglia's Children's Hospices, Cambridge, UK

\subsection{6/archdischild-2020-rcpch.305}

Introduction Paediatric palliative care (PPC) endeavours to provide holistic family-and-child-centred care, to children with life-limiting conditions (LLC). Advance care planning (ACP) is widely recommended and is believed to improve outcomes and experience. Practice varies widely within the UK, by geographical region as well as disease.

Aims To explore the experiences of bereaved parents and determine the main factors that influence the experiences of children who die of LLC and their families, including the impact of ACP.

Method This quantitative arm of a mixed-methods study used an anonymous questionnaire offered online and by post to all bereaved parents in the region whose children had died between December 2013 and June 2018.

The responses were analysed to determine the prevalence of ACP and determine factors that impact families' experiences - as measured by variables such as the availability of choice of place of death (PoD) - and various subjective perceptions of care.

Results Thirty questionnaires were returned. 53.3\% (16/30) of questionnaire respondents recalled a prognostic discussion at diagnosis - four out of ten families of children with cancer and seven out of nineteen with non-cancer diagnoses. $53.6 \%$ (15/28) reported ACP discussion. 10 reported 33.3 documented ACP.

Families who remembered ACP discussions were more likely to have been offered a choice of $\operatorname{PoD}(p=0.0107)$. Parents who reported access to a PPC team were more likely to report that they had been offered a choice of PoD $(p=0.01)$ and that the caring teams respected their wishes and desires $(p=0.05)$ than those who didn't. Parents' educational level, diagnosis of cancer or time elapsed between first prognostic discussion and death had no significant effect on ACP, choice of PoD or subjective experiences.

Conclusions Formal ACP and early prognostic discussions are not yet part of routine paediatric practice in the region. Formal ACP and early integration of palliative care may positively impact subjective experiences as well as outcomes such as realisation of preferred PoD.

Limitations lack of statistical power, diagnostic heterogeneity and socio-cultural homogeneity of the sample, potential recruitment and recall bias. Further research should explore a. impact of ACP in different patient groups and b. professionals' perceptions.

\section{G358}

\section{COST OF LONG TERM VENTILATION}

L Alkhalidi, R Belderbos, V Kumar. Paediatric Department, Royal Blackburn Hospital, Blackburn, UK

\subsection{6/archdischild-2020-rcpch.306}

Advances in Neonatal and Paediatric Intensive Care have increased the survival of children with life threatening or life limiting conditions. There has been a significant rise in children on long term home invasive ventilation.

High profile cases have been in the media recently with debate on whether such interventions should be implemented focussing on ethics but without evidence of cost benefit analysis.

Children on long term invasive ventilation are a high cost group with complex and varying underlying medical conditions requiring input from multiple teams, including 24 hour carers, medical and multidisciplinary team input as well as recurrent hospital and PICU admissions. In addition, the cost of equipment and drugs makes this a costly intervention.

In any limited healthcare system rationing decisions have to be made: drug and other therapies are subject to health economic analyses. This study aims to assess cost per annum for LTV and an cost benefit analysis.

Objectives

- Identify patients on LTV including comorbidities

- Assess cost of LTV to quantify cost-benefit analysis

- Measure outcomes : death/admissions/recovery

Methods

- Review of patients requiring home long term invasive ventilation July 2009 - July 2019

- Analysis of costs: clinic visits, hospital admissions, costs of equipment; cost of medication

- Outcomes and quality of life: mortality, admissions and length of stay; decannulation, ability to communicate and mobility analysis; ability of parents to work

Results

10 patients:

- 3 died (aged 1 year, 7 years and 15 years)

- 4 decannulated

- 3 ongoing LTV (aged 15 months, 19 and 22 years)

- 5 night package $£ 140,000$ pa

- accessories $£ 12,000$ pa

- replaceables/service $£ 92,000$

- average cost home LTV around $£ 350,000$ pa

Conclusions LTV ventilation is an expensive treatment: its use should be analysed on a cost benefit analysis in a similar way to other available treatments.

\section{G359 DIFFICULT DECISIONS IN PAEDIATRICS: SHARING, CONNECTING AND MANAGING ETHICAL TENSIONS IN PRACTICE}

P Coulson-Smith. Clinical Ethics and Law, Faculty of Medicine, University of Southampton, Southampton, UK

\subsection{6/archdischild-2020-rcpch.307}

Difficult Decisions in Paediatrics is a national forum which aims to share, connect and identify ethical issues in paediatrics. The forum was developed following a two part 